\title{
Magnetic nano-ordering in polycrystal and monocrystal chromium
}

\author{
T.Rozouvan, I.Yurgelevich, S.Rozouvan, L.Poperenko \\ Department of Physics, T.Shevchenko National University of Kyiv, \\ 4 Glushkova Ave., 03680 Kyiv, Ukraine
}

Received April 16, 2014

\begin{abstract}
Surface structures of polycrystalline and monocrystalline samples of chromium were investigated using the following techniques: magnetic force microscopy, scanning tunneling microscopy, and spectral ellipsometric method. Presence of magnetic nanostructure on the surface of polycrystalline chromium has been explained in terms of spin orientation of the atoms. Also we carried out numerical quantum mechanical calculations of chromium supercell and analyzed the results using experimentally obtained curves of the optical conductivity for chromium single crystal.
\end{abstract}

Поверхностная структура поликристаллического и монокристаллического хрома изучена с использованием магнитосиловой микроскопии, сканирующей туннельной микроскопии и спектральной эллипсометрии. Наличие магнитного наноструктурирования на поверхности поликристаллического хрома обьяснено ориентацией спинов атомов. Произведены численные квантовомеханические вычисления и сравнение с экспериментально полученными зависимостями оптической проводимости монокристаллического хрома.

Магнітне нано-упорядкування у полікристалічному і монокристалічному хрому. Т.Розуван, І.Юргелевич, С.Розуван, Л.Поперенко.

Поверхнева структура полікристалічного і монокристалічного хрому вивчалася 3 використанням магнітосилової мікроскопії, скануючої тунельної мікроскопії і спектральної еліпсометрії. Наявність магнітного наноструктурування на поверхні полікристалічного хрому пояснено орієнтацією спінів атомів. Проведено чисельні квантовомеханічні обчисления і порівняння з експериментально отриманими залежностями оптичної провідності монокристалічного хрому.

\section{Introduction}

Magnetic properties of macroscopic or microscopic chromium most of the times are related to antiferromagnetism. It is common knowledge that chromium is the most known for its' magnetic properties. It is the only elemental solid which shows antiferromagnetic ordering at the room temperature. In the case of chromium the magnetic properties are the results of spin-density-waves which make this $3 d$ metal's magnetism of more specific nature. Detailing of the current state of $\mathrm{Cr}$ magnetism studies is presented in profound reviews $[1,2]$. One of the most interesting trends in contemporary condensed matter physics of magnetic phenomenas is the study of materials on nanoscale level. Recent progress in experimental techniques, which deliver data about the samples' nanostructure, also allows the determining of material's magnetic nanoscale physical properties. For example, antiferromagnetism is explained in terms of imperfect nesting i.e. slightly different radii of electron- and hole-bands [3]. Spin fluctuations theory is applied to characterize dynamic magnetic succeptibilities of antiferromagnets [4], the alteration of $\mathrm{Cr}$ nanocrystals due to microstrain fluctuations and 


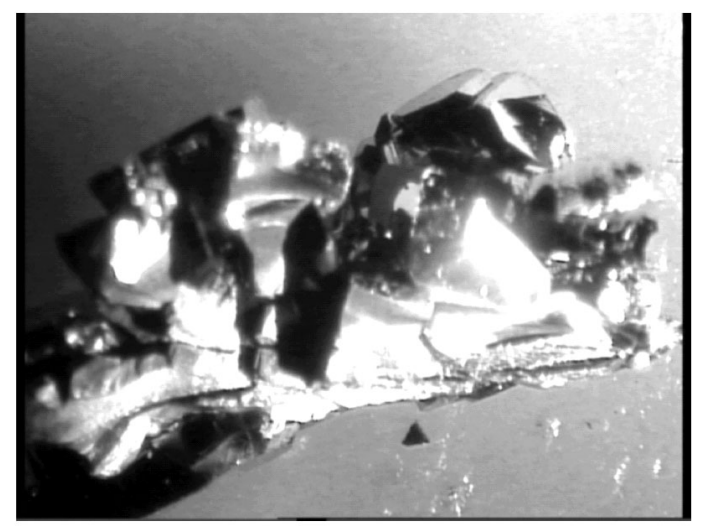

Fig. 1. Photo of chromium monocrystal. Vertical image size $5 \mathrm{~mm}$.

correlations with the crystallite sizes which were determined in [5]. Lower total magnetization of noncolinear ground magnetic states in small chromium clusters was studied in [6]. Magnetic force microscopy (MFM) and scanning tunneling microscopy (STM) are among basic techniques to study $\mathrm{Cr}$ surface nanomagnetism. The techniques allow to check the growth, thickness, composition and structure of oxide thin films on the $\mathrm{Cr}$ surface [7] and to detect $\mathrm{C}, \mathrm{N}$ and $\mathrm{O}$ impurities on $\mathrm{Cr}(100)$ single crystal surface [8]. Oxygen integrated into the $\mathrm{Cr}$ lattice induces ferromagnetic order and no variation of magnetic properties if oxygen is adsorbed on top of the surface. No indication of ferromagnetism of the oxygen-free surface has been observed [9]. Surface nanomagnetism can be a topic practical interest due to its possible application in magnetic sensors, spin electronics, and magnetic recording media. The goal of this article is to study the magnetic properties of chromium surface on nanoscale level by applying precise MFM and STM techniques.

\section{Experimental}

In this work we studied samples of polycrystalline and monocrystalline chromium. Chromium monoctystal (see photo in Fig. 1) was grown using the Czochralski process. Small size of the crystal and its irregular geometric shape created certain difficulties while applying atomic force microscopy and spectroscopic ellipsometry methods. In Fig. 1 the sample has ideal flat faces, although its small size creates experimental difficulties, especially while fixating it and with the precision alignment of the faces orientation. Since atomic force microscopy (AFM) is a robust technique for surface profile measurements, first we checked the roughness of



Fig. 2. AFM scan of chromium polycrystalline polished surface.

our polycrystalline chromium sample (Fig. 2) applying AFM. We used microscope INTEGRA NT-MDT, which allowed us to conduct measurements in atomic force microscopy and tunneling microscopy regimes. In our case the latter was used for precise surface topology study. Atomic force microscopy measurements have been conducted both in contact and semicontact regimes. Spatial resolution at these measurements was determined by a cantilever tip curvature and reached $40 \mathrm{~nm}$ in horizontal direction. Scanning tunneling microscopy spatial resolution reached up to $1 \mathrm{~nm}$. In Fig. 2 there are visible polished surfaces of the sample which have the roughness in the range of approximately ten nanometres. The presence of vertical lines on the scanned surfaces is typical since they contain fine particles of the cleaved material, which will inevitably appear with mechanical polishing. To study chromium's magnetic properties we used magnetic force microscopy. It consists of two passes of the needle, where the first pass is when the system determines the profile of the surface (Fig. 3, on the right). During the second pass the needle, which is covered in ferromagnetic material (cobalt), is held at a predetermined height (in our case it's $50 \mathrm{~nm}$, which significantly exceeds the size of the roughness of the sample) and repeats the motion profile of the measured surface of the first pass. This approach allows us to measure the distribution of only the magnetic component of the force and to exclude the short-distance van der Waals force and as a result to eliminate the presence of systematic measurements error. The magnetic force is the force which arises be- 

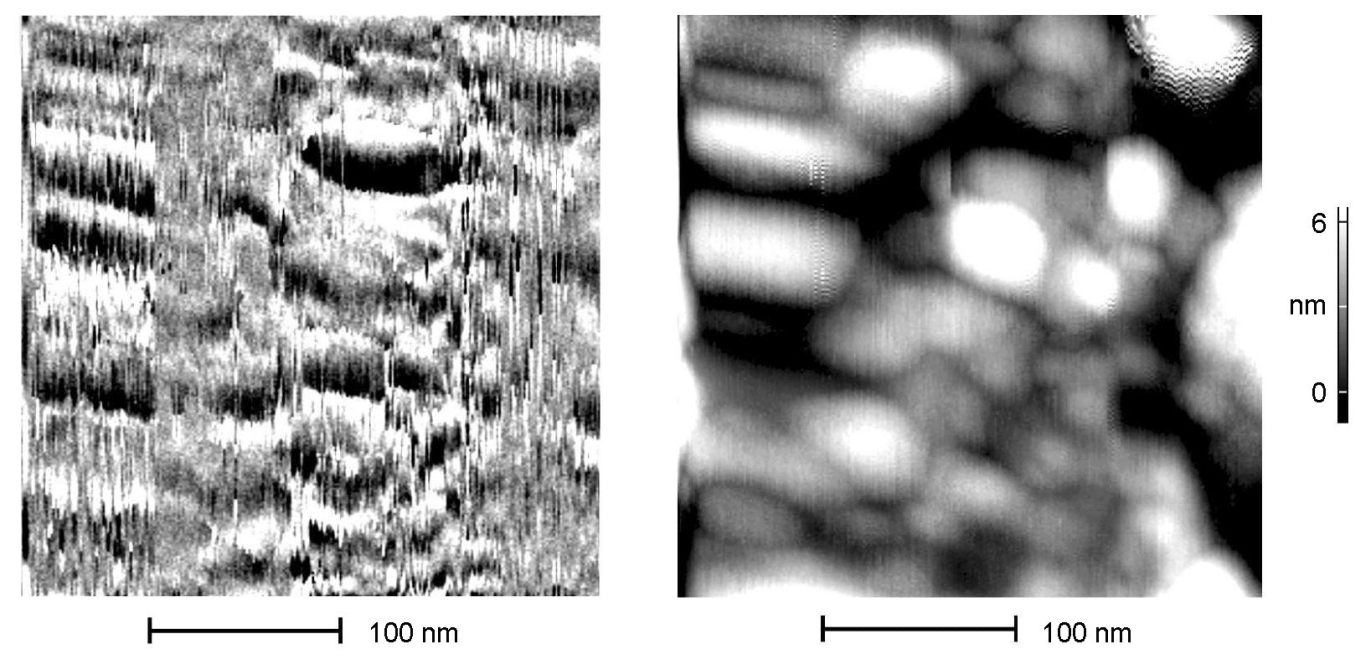

Fig. 3. Magnetic force microscopy results of chromium polycrystal: magnetic force distribution (left), AFM scan surface (right).
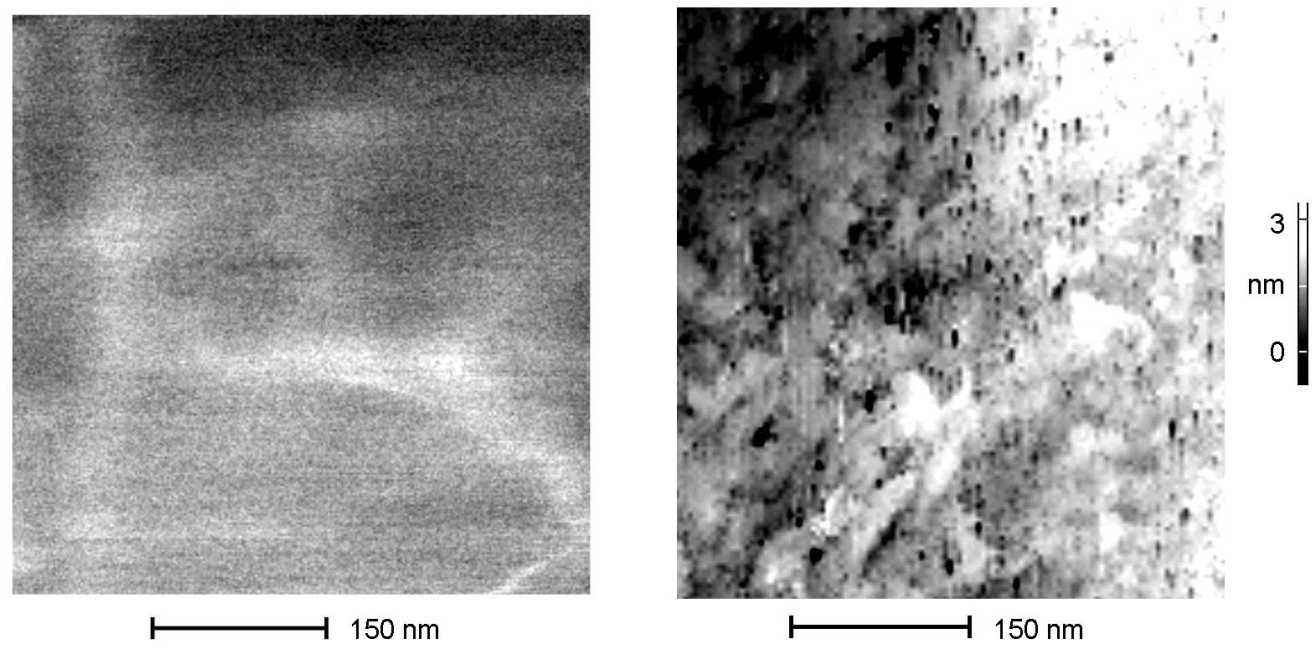

Fig. 4. Magnetic force microscopy of chromium monocrystal: magnetic force distribution (left), STM scan surface (right), spatial resolution $1 \mathrm{~nm}$.

tween the ferromagnetic tip of the cantilever and the substrate. In Fig. 3 there are visible distributions of the magnetic force component of the scanned surface, which is not constant (Fig. 3, on the left) which can be an indication of the heterogeneousness of the magnetic nanostructure on chrome's surface. Profiles from Fig. 3 on the left and right do not match which strongly indicates the presence of magnetic related nanopatterns of the sample surface. Findings of magnetic force microscopy of the surface of the monocrystal chrome are presented in Fig. 4. Unlike the data in Fig. 3, profiles represented a permanent constant within the margins of measurement error. Measurements are impossible on a very smooth plane surfaces (e.g. atomic plane) since the radius of the tip of the cantilever's needle exceeds $30 \mathrm{~nm}$. It is too low spatial resolu- tion, when surface roughness is a few nanometers. Due to these additional factors we also performed measurements using high spatial-resolution scanning tunneling $\mathrm{mi}$ croscopy with the results which are presented on the right in Fig. 4. Sizes of the irregularities of the monocrystalline chrome were determined to be in the range of several nanometres. Availability of magnetic nanostructures in polycrystalline chrome may be explained as the influence of surface structure and irregularities between separate monocrystals of polycrystalline substrate. By this approach the images that for monocrystalline Fig. 4 chromium contain distortions in the surface's homogenous magnetic structure. 


\section{DFT calculations and discussion}

As we know from theoretical predictions [10] (001) surface of $\mathrm{Cr}$ can have three different spin arrangements-ferromagnetism, inside-the-surface $\quad c(2 \times 2)$ antiferromagnetism, and "topological" antiferromagnetism between ferromagnetic terraces separated by single steps. A majority-spin surface state with a binding energy of $1 \mathrm{eV}$ at the $\Gamma^{-}$point and ferromagnetic $\mathrm{Cr}(001)$ surface associated magnetic moment of $2.49 \mu \mathrm{B}$ was found [11]. A surface magnetic phase transition on $\mathrm{Cr}(100)$ at transition temperature of $780 \pm 50 \mathrm{~K}$ was also observed [12]. A possible theoretical explanation of our MFM, STM experimental data can be based on $\mathrm{Cr}$ surface spins nanoscale topology. Impurities seem not to be responsible for surface nanomagnetic fluctuations. If we had point defects (interstitial impurity atoms or substitutional atom) it would not be registered in our measurements because of its non-atomic spatial resolution $(40 \mathrm{~nm}$ for AFM and $1 \mathrm{~nm}$ for STM). The clusters of impurities in $\mathrm{Cr}$ sample would form domains on surface with sharp boundaries $[8,13]$ and as a consequence would result in distortion of optical conductivity dispersion curves measured earlier [14]. We completed numerical simulations of chrome supercell using density functional theory (DFT) to model the process of magnetic nanostructuring in polycrystalline chrome. In order to find approximate solutions of the Schrodinger equation for crystal lattice supercells the whole problem can be reduced to a more solvable case by applying the charge density $n(r)$ variable. DFT can formally be regarded as a development of the Thomas Fermi theory $[15,16]$ and the self-consistent Hartree equations [17]. The DFT equations are based on developed Hohenberg-Kohn theorem [18] and Kohn-Sham system [19]. The respective Kohn-Sham equations for the effective single-particle wave functions can be written in the form

$$
\begin{gathered}
{\left[-\left(\hbar^{2} / 2 m\right) \Delta+V_{e f f}\right] \Phi_{i}(r)=E_{i} \Phi_{i}(r),} \\
V_{e f f}(r)=V(r)+\int \frac{e^{2} n\left(r^{\prime}\right)}{\left|r-r^{\prime}\right|} d r^{\prime}+V_{X C}(r), \\
n(r)=\sum_{i=1}^{N}\left|\Phi_{i}(r)\right|^{2},
\end{gathered}
$$

where the local exchange-correlation potential $V_{X C}(r)$ is the partial derivative of the

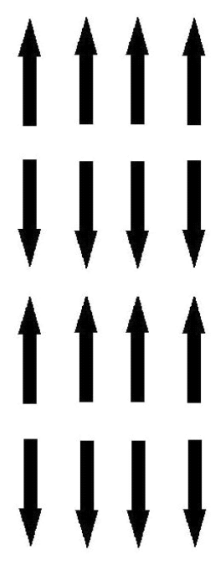

a)

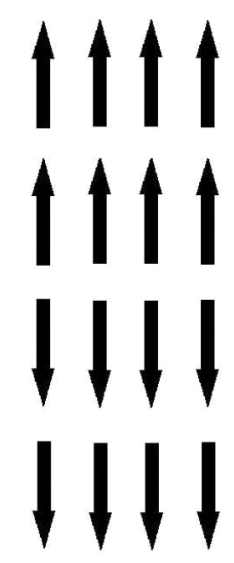

b)
Fig. 5. Two spin orientation maps used for numerical simulation.

exchange correlation functional $E_{X C}(n(r))$ with respect to the charge density $n(r)$ for the ground-state $n_{0}(r)$. The first term in the first equation (1) corresponds to the kinetic energy of non-interacting electrons and the second term of the second equation (1) is just the Hartree contribution $V_{H}(r)$ to the interaction energy. In order to make numerical simulations with non-collinear DFT (taking into account electron spins) we add an additional member to Hamiltonian in Eq. (1) $\pm \mu_{B} B_{X C, Y}(r)\left(B_{X C, Y}(r)\right.$ is magnetic field, $\mu_{B}$ is Bohr magneton) for wave functions and $\Phi_{i}(r, \uparrow)$ and $\Phi_{i}(r, \downarrow)$ (for electrons with spins up and down oriented along Y axis) [20].

The different irregularities in chromium crystal lattice (e.g. terraces on the surface) and as a result irregularities in spin patterning can be modeled by two different sets of spin-orientation displayed in Fig. 5. In order to determine the types of influences of these spin orientation on chromium's electronic density and energy parameters we used ABINIT software in LINUX environment to perform DFT calculations. In Fig. 6 we presented a typical distribution of the electronic density for the chosen chrome supercell. Calculation findings for two types of spin orientation from Fig. 5 show that differences in spatial distribution of electronic density in Fig. 6 are insignificant.

Distributions of electron density of states for two types of spin patterns from Fig. 5 are shown in Fig. 7 and demonstrate noticeable differences in the two curves. Practically this means that non-flat profile of the surface, which leads to uneven boundary conditions for surface terraces or 


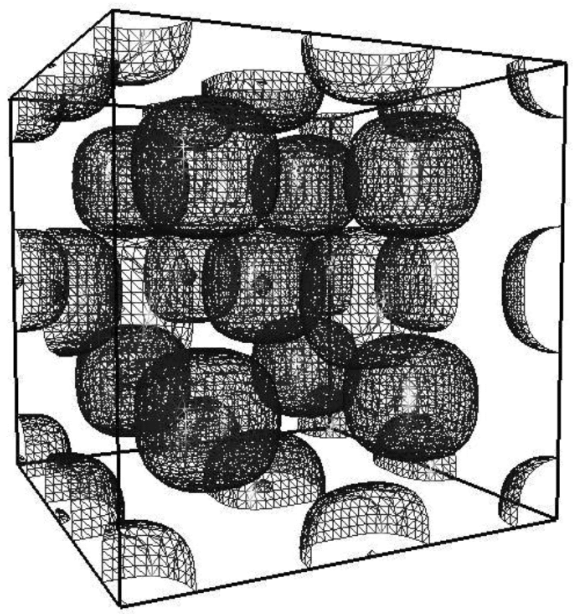

Fig. 6. Calculated $\mathrm{Cr}$ electron density for $2 \times 2 \times 2$ supercell the Fig. $5 b$ orientation map. Density isovalue 0.045 . Size of faces of the cube $0.661 \mathrm{~nm}$.

neighboring crystallites (thus it leads to changes in the distribution curves of electron density of states) can result in nanostructuring of the spin orientation in neighboring nanoclustered surfaces. In our case the spin orientation from Fig. 5,b is more energetically favorable comparing to the regular spin set from Fig. 5, a.

Spectral ellipsometric measurements performed on a single crystal of chromium, with results shown in Fig. 8, provide indirect confirmation of the accuracy of our numerical simulations. Since spectral dependence of optical conductivity is the convolution of the distribution of the electronic states [21], similarity of the curve drawings in Fig. 7 and Fig. 8 is collateral proof of the accuracy of our calculations. The shape of the curve in Fig. 8 is similar to the results of the experiments in [14] which were acquired for grinded polycrystalline surface.

\section{Conclusions}

Experiments on monocrystalline and polycrystalline chromium using magnetic force and scanning tunneling microscopy demonstrated that nanoscale magnetic force spatial distributions can have certain peculiarities. Polycrystalline chrome displayed heterogeneuos magnetic field distribution along the surface. This may be explained by taking into account boundary conditions between the irregularities. On the surface of the polycrystalline chromium the neighboring spins in spots between crystallites or terraces may have disruptions in antiferromagnetic order. As a result the orientation

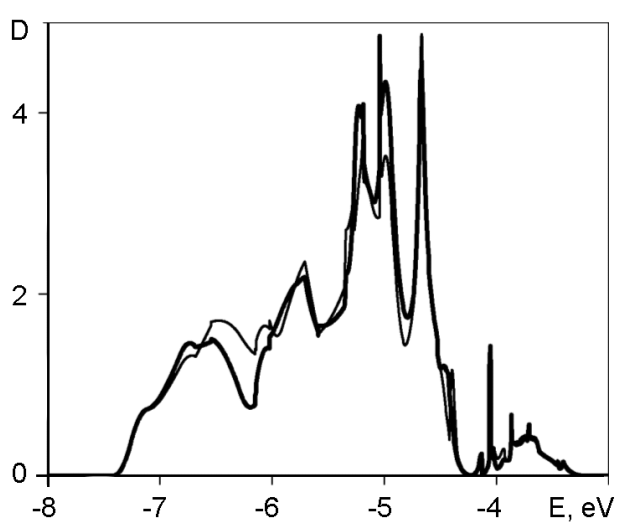

Fig. 7. Calculation of density of states $(D)$ for spin map $a$ (thick line) and $b$ (thin line).

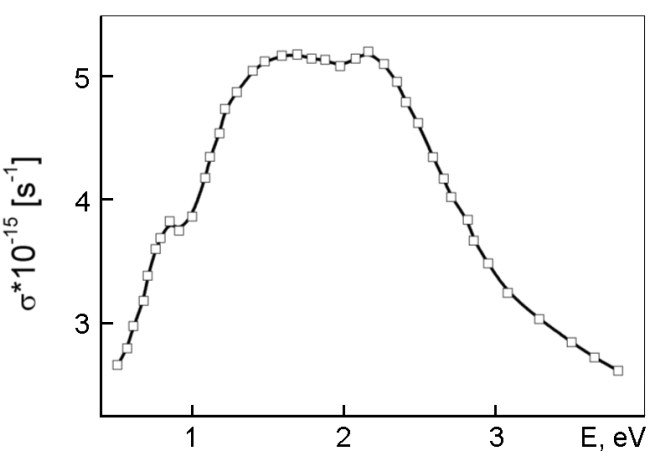

Fig. 8. Optical conductivity of $\mathrm{Cr}$ monocrystal obtained by spectral ellipsometry.

of the chrome spins in the vicinity of the surface irregularities can change its direction to parallel. Numerical calculations conducted by the method of density functional theory using the software package "ABINIT" for chromium supercells resulted in different electron density of states curves for different spin patterning. As a consequence of the numerical simulations, a non antiferromagnetic order may be more energetic favorable in some spots of the chrome surface. Spectral ellipsometry data confirm nanoscale character of the observed effects. Antiferromagnetic ordering effects in chromium may play an important role in construction of novel nanodevices.

\section{References}

1. Eric Fawcett, Rev.Modern Phys., 60, 209 (1988).

2. C.Herring, Exchange Interactions Among Itinerant Electrons, Vol. IV of Magnetism, ed. by G.Rado and H.Suhl, Academic, New York (1966).

3. A.L.Rakhmanov, A.V.Rozhkov, A.O.Sboychakov, Franco Nori, Phys.Rev. B, 87, 075128 (2013). 
4. J.B.Staunton, J.Poulter, B.Ginatempo et al., Phys. Rev. B, 62, 1075 (2000).

5. D.Wardecki, R.Przenioslo, M.Bukowski et al., Phys. Rev. B, 86, 064410 (2012).

6. C.Kohl, G.F.Bertsch, Phys.Rev.B, 60, 4205 (1999).

7. V.Maurice, S.Cadot, P.Marcus, Surf.Sci., 458, 195 (2000).

8. M.Schmid, M.Pinczolits, W.Hebenstreit, P.Varga, Surf. Sci., 377, 1023 (1997).

9. F.Meier, D.Pescia, T.Schriber, Phys. Rev.Lett., 48, 645 (1982)

10. S.Blugel, D.Pescia, P.H.Dederichs, Phys. Rev. $B, 39,1392$ (1989).

11. C.L.Fu, A.J.Freeman, Phys.Rev.B, 33, 1755 (1986).

12. L.E.Klebanoff, S.W.Robey, G.Liu, D.A.Shirley, Phys. Rev. B, 30, 1048 (1984).
13. Lee Jookyung, Ph.D. Dissertation, University of Maryland (2010).

14. V.D.Karpusha, L.V.Poperenko, I.A.Shaikevich, Fizika, Himiya, Mehanika, 9, 118 (1991).

15. L.H.Thomas, Proc.Cambridge Philos. Soc., 23, 542 (1927).

16. E.Fermi, Atti Accad.Naz.Lincei, Cl.Sci.Fis. Mat.Nat.Rend., 6, 602 (1927).

17. D.R.Hartree, Proc.Cambridge Philos.Soc., 24, 89 (1928).

18. P.Hohenberg, W.Kohn, Phys. Rev., 136, B864 (1964).

19. W.Kohn, L.J.Sham, Phys.Rev., 140, A1133 (1965).

20. B.H.Bransden, C.J.Joachain, Physics of Atoms and Molecules, Prentice Hall (2003).

21. C.N.Berglund, W.E.Spicer, Phys.Rev., 136, 1030 (1964). 